\title{
Resource Allocation -WiMAX Systems
}

\author{
Dr. P. Satish Kumar \\ Dept of ECE,MLR Institute of Technology \\ Dundigal, Hyderabad, RR dist, AP. India \\ R MURALI PRASAD \\ Dept of ECE, LR Institute of Technology \\ Dundigal, Hyderabad, RR dist, AP. India
}

\begin{abstract}
Resource allocation is been shown to provide substantial performance gain in wireless systems, such as WiMAX, when full channel state information (CSI) is available. But in some fading environments (e.g., fast fading), there may not be a feedback link. In this paper, we consider resource allocation strategies for downlink multiuser mobileWiMAX systems. We address the problem of subchannel assignment and power allocation. This problem is formulated as a nonlinear stochastic constrained optimization problem. We provide an analytical solution based on the Lagrange.
\end{abstract}

Keywords: Resoure allocation, wireless system, WiMAX system

\section{Introduction}

The mobile version of the Worldwide Interoperability for Microwave Access (mobile WiMAX) is one of the solutions in the competition for wireless broadband applications in challenging mobile environments (WiMAX Forum, 2006)(WiMAX Forum, 2007). One of the future aspects of OFDMA is the Sub channelization which allows to group a total number of subcarriers into subsets of subcarriers called sub channels (S. Pietrzyk, 2006).

Mobile WiMAX aimed at delivering broadband mobile services ranging from real-time interactive gaming, VoIP, and streaming media to non real-time.

Most of the previous works on OFDMA resource allocation have considered only the case where instantaneous channel state (CSI) is available at the transmitter and various algorithms based on instantaneous CSI have been developed (C. Y. Wong, C. Y. Tsui, R. S. Cheng and K. B. Letaief, 1999)(M. Ergen, S. Coleri and P. Varaiya, 2003)(D. Kivanc, G. Li and H. Liu, 2003)(N. Damji and T. Le-Ngoc, 2006)(T. Sartenaer, L. Vandendorpe and J. Louveaux, 2005)(T. Sartenaer, J. Louveaux and L, 2008)(I. C. Wong, Z. Shen, B. L. Evans, and J. G. Andrews, 2004)( X. Zhang, E. Zhou, R. Zhu, S. Liu, and W. Wang, 2005)(Z. Han, Z. Ji, and K. J. R. Liu, 2005)(Y. Yao and G. B. Giannakis, 2005)(F. Brah, L. Vandendorpe, and J. Louveaux, 2008). Following the Hungarian approach, (M. Ergen, S. Coleri and P. Varaiya, 2003) proposed an iterative algorithm for power minimization and bit loading. To reduce the computational complexity, (D. Kivanc, G. Li and H. Liu, 2003) proposed low complexity and computationally efficient bandwidth and power allocation algorithms to solve the problem of minimizing the total power consumption under bit error rate and transmission rate constraints. In (N. Damji and T. Le-Ngoc, 2006), the performance of bandwidth-constrained power minimization and power minimization schemes in terms of outage probability. Fairness issues in a wire line multi-access channel have been taken into account in (T. Sartenaer, L. Vandendorpe and J. Louveaux, 2005)(T. Sartenaer, J. Louveaux and L, 2008). The concept of balanced capacity to characterize the multiuser channel performance with total power constraints in (T. Sartenaer, L. Vandendorpe and J. Louveaux, 2005) and they extend the concept to individual power constraints in (T. Sartenaer, J. Louveaux and L, 2008). This concept of balanced capacity is closely related to the one presented in (I. C. Wong, Z. Shen, B. L. Evans, and J. G. Andrews, 2004). In (X. Zhang, E. Zhou, R. Zhu, S. Liu, and W. Wang, 2005), suboptimal resource grids and power allocation algorithms to maximize the total throughput under user's data rate requirement are presented. Rate-power allocation algorithms for expected mutual information maximization based on partial channel knowledge have been developed in (Y. Yao and G. B. Giannakis, 2005). In (F. Brah, L. Vandendorpe, and J. Louveaux, 2008), the authors investigated the impact of imperfect channel information on OFDMA-based systems. Power allocation for argotic capacity maximization in relay networks based on CDIT under high SNR regime has been studied in (C. T. K. Ng and A. J. Goldsmith, 2006).

The remainder of this paper is organized as follows. In Section 2, the system model considered is described and the argotic weighted-sum rate is derived. The problem of multiuser resource allocation based on CDIT is 
formulated in Section 3 and a solution guideline is given. In Section 4, some simulation results are presented. Finally, conclusions are drawn in Section 5. Mobile WiMAX while satisfying user's specific minimum rate demand and system fairness requirement for a given power budget. Though this is originally a nonlinear optimization problem, the problem can be reformulated as a Lagrangian dual problem. From this, a method has been proposed efficiently to solve the problem. The proposed method can find the optimal solution with significant lower computational complexity than the optimal CSIT-based allocation schemes. In fading environments, even with CDIT only, adaptive resource allocation strategies provide performance gain for OFDMA systems. Since user mobility is the principal driving force for mobile WiMAX,CDIT-based resource allocation

Strategies are of particular interest. These methods can be applied to other mobile OFDMA-based wireless systems such as Long Term Evolution (LTE) or High-Speed Downlink Packet Access (HSDPA).

\section{System Model}

We consider a single cell downlink WiMAX communication from a base station (BS) to $K$ mobile user terminals, over a realistic frequency-selective fast fading channel with total bandwidth $B$. The BS splits up the downlink bandwidth into different sub channels. Hence, the base station has only channel distribution information (CDI), but no knowledge of the instantaneous channel realizations. Assuming that the receiver employs a maximum ratio combiner (MRC), the effective $S N R$ of user $k$ at $m$ th subchannel is given by

$$
\gamma_{k, m}=\frac{1}{\Gamma_{k} N \sigma_{n}^{2}} \sum_{n=0}^{N-1} g_{k, m}(n) .
$$

In (1), $N$ is the number of distributed subcarriers per- subchannel, $g(n)$ is the channel gain of user $k$ at subcarrier $n$ of $m$ th subchannel, which is the product of the distance attenuation and the fast fading gain, $\sigma_{n}{ }^{2}$ is the noise variance, $\Gamma_{k}$ is referred to as SNR gap related to the required bit error rate of user $k(\mathrm{BER} k)$ and is approximated as $\Gamma_{k} .-\operatorname{in}\left(5 \mathrm{BER}_{k} / 1.5\right)$ for QAM modulations [10]. We assume a Rayleigh channel model. Hence $\gamma$, is a central chi-squared $\left(\chi^{2}\right)$ distributed random variable with two degrees of freedom and with probability density function (pdf)

$$
T_{\gamma_{k, m}}\left(\gamma_{k, m}\right)=\frac{1}{\bar{\gamma}_{k, m}} e^{-\gamma_{k, m} / \bar{\gamma}_{k, m}}
$$

Where $\bar{\gamma}_{k, m}$ is the means of $\gamma_{k, m}$ distribution

Each user is adaptively assigned a number of different Sub channels to send and receive data. An indicator $\rho_{k, m}$ issued to represent whether the $m$ th subchannel is assigned to user $k$. Note that in a single cell OFDMA system, each sub channel can be assigned to at most one user at any time, that is $\sum_{k=1}^{K} \rho_{k, m} \in 0,1$ for all $m$. Due to the consideration for the reduction of the signaling overhead in WIMAX, the power is equally distributed across subcarriers within each sub channel. We assume the duration of the transmission codeword's is long enough to undergo all channel realizations. We further assume perfect CDIT, thereby allowing to take the expectation over the distribution. The argotic weighted sum rate of the multi user system is defined as

$$
U_{\gamma}=E_{\gamma} \sum_{k=1}^{K} \frac{1}{\bar{R}_{k}^{\alpha_{f}}} \sum_{m=1}^{M} \rho_{k, m} \log _{2}\left(1+p_{k, m} \gamma_{k, m}\right),
$$

where

$\gamma=\left[\gamma^{T}, \ldots, \gamma^{T}\right]$ with $\gamma_{k}=\left[\gamma, \ldots, \gamma, \gamma_{k, M}\right] \mathrm{Pk}, \mathrm{m}$ denotes the power allocated to the user $k$ on subchannel $m$, $E\{$.$\} represents the statistical expectation with respect to \gamma, R_{k}$ is user $k$ 's average data rate so far at the allocation time, and $\alpha_{f}$ is a tunable fairness parameter. Setting $\alpha_{f}$ to 1 results in the proportional fair allocation. For $\alpha_{\text {. } f}$ 0 , this results in maximum throughput allocation. The average user rates $R_{k}$ are update according to

$$
\bar{R}_{k}(t+1)=1-\frac{1}{\tau_{c}} \bar{R}_{k}(t)+\frac{1}{\tau_{c}} r_{k}(t)
$$

where $r_{k}(t)$ is the rate allocated to user $k$ at time $t$ and $\tau_{c}$ is the parameter that controls the latency of the system. 
This way, we consider both current rate as well as rates given to the users in the past, what is suitable for long-term fairness evaluation.

\section{CDIT-Based Constrained Resource Allocation}

\subsection{Formulation of the Problem}

The issue is how to adaptively assign the $M$ sub channels to the $K$ users and distribute

The total power budget $P_{\text {tot }}$ in order to maximize the argotic weighted-sum rate (3) while satisfying user's minimum rate and system fairness requirements under a total power constraint. Mathematically, this constrained optimization

$$
\begin{aligned}
& f^{*}=\max _{\rho_{k, m}, p_{k, m}} E_{\gamma} \sum_{k=1}^{K} \frac{1}{\bar{R}_{k}^{\alpha_{j}}} \sum_{m=1}^{M} \rho_{k, m} \log _{2}\left(1+p_{k, m} \gamma_{k, m}\right) \\
& \text { subject to } \\
& E_{\gamma} \sum_{m=1}^{M} \rho_{k, m} \log _{2}\left(1+p_{k, m} \gamma_{k, m}\right) \geq R_{k}, \\
& E_{\gamma} \sum_{k=1}^{K} \sum_{m=1}^{M} \rho_{k, m} p_{k, m} \leq P_{\text {tot }} .
\end{aligned}
$$

The first constraint in (5) is for the user's specific minimum data rate demand. We assume that appropriate admission control is performed such that the minimum data rates $R_{k}$ are feasible. The second constraint is system limitation on transmits powers.

Note that the optimization problem (5) involves both continuous variables $p$, and Boolean variable $\rho$ Such an optimization problem is neither convex nor concave with

Respect to $\rho k m, P k, m)$

\subsection{Solution Based on Lagrange Dual Decomposition}

We can solve problem (5) using the Lagrange dual decomposition

Framework. Following the approach in (Z. Han, Z. Ji, and K. J. R. Liu, 2005), we relax $\rho_{\mathrm{k}, \mathrm{m}}$ to be $\rho k, m \in[0$, 1]. Then $\rho$, can be regarded as time-sharing factor. Thanks to the linearity property of the expectation, the Lagrangian function of the primal problem (5) can be expressed as

$$
\begin{aligned}
L_{\gamma}\left(p_{k, m}, \rho_{k, m}, \lambda_{k}, \mu\right) & \\
= & \sum_{k=1}^{K} \sum_{m=1}^{M} E_{\gamma_{k, m}} \frac{\rho_{k, m}}{\bar{R}_{k}^{\alpha_{f}}} \log _{2}\left(1+p_{k, m} \gamma_{k, m}\right) \\
& +\sum_{k=1}^{K} \lambda_{k} \sum_{m=1}^{M} E_{\gamma_{k, m}}\left\{\rho_{k, m} \log _{2}\left(1+p_{k, m} \gamma_{k, m}\right)\right\} \\
& -\mu \sum_{k=1}^{K} \sum_{m=1}^{M} \rho_{k, m} p_{k, m}-\sum_{k=1}^{K} \lambda_{k} R_{k}+\mu P_{\mathrm{tot}},
\end{aligned}
$$

Where $\lambda_{k}$ and $\mu$ are Lagrangian multipliers. Let $p_{k, m}^{*}$ and $\rho_{k, m}^{*}$ denote the optimal solution of (6). We first investigate the problem for fixed values of $\lambda_{k}$ and $\mu$. By Karush-Kuhn-Tucker (KKT) first optimality condition (S. Boyd and L. Vandenberg he, 2004), $p_{k, m}^{*}$ and $\rho_{k, m}^{*}$ should satisfy the following:

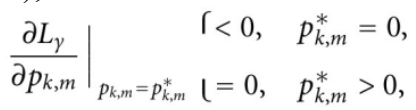

$$
\begin{aligned}
& \left.\frac{\partial L_{\gamma}}{\partial \rho_{k, m}}\right|_{\rho_{k, m}=\rho_{k, m}^{*}} \begin{array}{ll}
<0, & \rho_{k, m}^{*}=0, \\
=0, & 0<\rho_{k, m}^{*}<1, \\
>0, & \rho_{k, m}^{*}=1 .
\end{array}
\end{aligned}
$$

For a nonzero power allocation and $\rho_{k, m}^{*} \in(0,1)$, obtain from (7) and (8) 


$$
\begin{array}{r}
\rho_{k, m}^{*} E_{\gamma_{k, m}} \frac{\bar{R}_{k}^{\left(-\alpha_{f}\right)}+\lambda_{k}}{\ln 2} \cdot \frac{\gamma_{k, m}}{1+p_{k, m}^{*} \gamma_{k, m}}-\mu=0, \\
E_{\gamma_{k, m}} \frac{\bar{R}_{k}^{\left(-\alpha_{f}\right)}+\lambda_{k}}{\ln 2} \cdot \ln \left(1+p_{k, m}^{*} \gamma_{k, m}\right)-\mu p_{k, m}^{*}=0 .
\end{array}
$$

We deduce from (9) that $p_{k, m}^{*}$ has to satify the following condition

$$
\rho_{k, m}^{*} E_{\gamma_{k, m}} \frac{\gamma_{k, m}}{1+p_{k, m}^{*} \gamma_{k, m}}-\frac{\mu \ln 2}{A_{k}}=0,
$$

where $A_{k}=\bar{R}_{k}^{\left(-\alpha_{f}\right)}+\lambda_{k}$. the value of $\mathrm{Pk}, \mathrm{m}$ is undefined, and any value can be taken without any infulences on the objective function or on the constraints. On the other hand, for any

Other positive value, $\rho_{\mathrm{k}, \mathrm{m}}$ vanishes out of the expression and

We get

$$
E_{\gamma_{k, m}} \frac{\gamma_{k, m}}{1+p_{k, m}^{*} \gamma_{k, m}}-\frac{\mu \ln 2}{A_{k}}=0 .
$$

We can use the pdf of the SNR distribution (2) to transform (12) into

$$
\int_{0}^{\infty} \frac{\gamma_{k, m}}{1+p_{k, m}^{*} \gamma_{k, m}}-\frac{\mu \ln 2}{A_{k}} \cdot \frac{1}{\bar{\gamma}_{k, m}} e^{-\gamma_{k, m} / \bar{\gamma}_{k, m}} d \gamma_{k, m}=0
$$

Which is equalent to

$$
\begin{aligned}
& \frac{\mu \ln 2}{A_{k}} p_{k, m}^{* 2} \bar{\gamma}_{k, m}-p_{k, m}^{*} \bar{\gamma}_{k, m} \\
& +\exp \frac{1}{p_{k, m}^{*} \bar{\gamma}_{k, m}} E_{1} \frac{1}{p_{k, m}^{*} \bar{\gamma}_{k, m}}=0,
\end{aligned}
$$

where

$$
E_{1}(x)={ }_{1}^{\infty} \frac{e^{-t x}}{t} d t
$$

Is the exponential integral function of $\mathrm{x}(\mathrm{M}$. Abramowitz and I. Stegun, 1964),

Equation (10) is equivalent to

$$
E_{\gamma_{k, m}} \frac{A_{k}}{\mu \ln 2} \ln \left(1+p_{k, m}^{*} \gamma_{k, m}\right)-p_{k, m}^{*}=0 .
$$

Using the pdf (2), (16) can be transformed into

$$
\begin{gathered}
\int_{0}^{\infty} \frac{A_{k}}{\mu \ln 2} \ln \left(1+p_{k, m}^{*} \gamma_{k, m}\right)-p_{k, m}^{*} \\
\cdot \frac{1}{\bar{\gamma}_{k, m}} e^{-\gamma_{k, m} / \bar{\gamma}_{k, m}} d \gamma_{k, m}=0,
\end{gathered}
$$

Which is finally equivalent to

$$
\frac{A_{k}}{\mu \ln 2} \exp \frac{1}{p_{k, m}^{*} \bar{\gamma}_{k, m}} E_{1} \frac{1}{p_{k, m}^{*} \bar{\gamma}_{k, m}}-p_{k, m}^{*}=0 .
$$

From (14) and (18), we derive

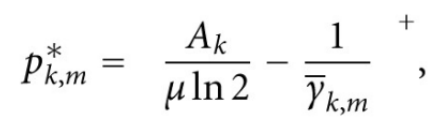

We have from (8) and (18) that

$$
\rho_{k, m}^{*}= \begin{cases}1, & \text { if } G_{k, m}\left(p_{k, m}^{*}\right)>0, \\ 0, & \text { if } G_{k, m}\left(p_{k, m}^{*}\right)<0,\end{cases}
$$


Where

$G_{k, m}\left(p_{k, m}^{*}\right)=\frac{A_{k}}{\mu \ln 2} \exp \frac{1}{p_{k, m}^{*} \bar{\gamma}_{k, m}} E_{1} \frac{1}{p_{k, m}^{*} \bar{\gamma}_{k, m}}-p_{k, m}^{*}$

Due to the excusive subchannel assignment constraint in OFDMA, we can conclude that for each subchannel m, if $\mathrm{Gk}, \mathrm{m}$ are all different, then only the user with the largest $\mathrm{Gk}, \mathrm{m}$ can use that subchannel, in other wrds,

$$
\rho_{k_{m}^{*}, m}^{*}=1, \quad \rho_{k, m}^{*}=0, \quad \forall k \neq k_{m}^{*},
$$

Where

$$
k_{m}^{*}=\arg \max _{k} G_{k, m}\left(p_{k, m}^{*}\right)
$$

As Substituting (19) into the Lagrange function (6) and thanks to the exclusive subchannel assignment constraint, we obtain the following per-subchannel dual problem:

$$
g^{*}=\min _{\lambda_{k}, \mu} L_{\gamma}^{*}\left(\lambda_{k}, \mu\right)
$$

where $L_{\gamma}^{*}\left(\lambda_{k}, \mu\right)$ is the dual function given by

$$
\begin{aligned}
L_{\gamma}^{*}\left(\lambda_{k}, \mu\right)= & \left.E_{\gamma_{k, m}}{ }^{\prime} \bar{R}_{k}^{\left(-\alpha_{f}\right)} \log _{2} \frac{\bar{R}_{k}^{\left(-\alpha_{f}\right)}+\lambda_{k}}{\mu \ln 2} \bar{\gamma}_{k, m}\right) \\
& +\lambda_{k} E_{\gamma_{k, m}}{ }^{\prime} \log _{2} \frac{\bar{R}_{k}^{\left(-\alpha_{f}\right)}+\lambda_{k}}{\mu \ln 2} \bar{\gamma}_{k, m} \mathrm{~J} \\
& -\lambda_{k} R_{k}-\mu \frac{\bar{R}_{k}^{\left(-\alpha_{f}\right)}+\lambda_{k}}{\mu \ln 2}-\frac{1}{\bar{\gamma}_{k, m}}+\mu P_{\text {tot }} .
\end{aligned}
$$

Next we turn to the optimization of the dual function (25) over $\mu$ and $\lambda$. First we consider the optimization over $\lambda$ for $\mu$ fixed to find. We differentiate (25) with respect to $\lambda_{k}$ and set the derivative to 0 to obtain

$$
\left.\frac{\partial L_{\gamma}^{*}}{\partial \lambda_{k}}\right|_{\lambda_{k}=\lambda_{k}^{*}}=\log _{2} \frac{\bar{R}_{k}^{\left(-\alpha_{f}\right)}+\lambda_{k}}{\mu \ln 2} \bar{\gamma}_{k, m}-R_{k}=0 .
$$

The optimum $\lambda_{k}^{*}$ is derived from (26) as follows:

$$
\lambda_{k}^{*}(\mu)=2^{R_{k}} \frac{\mu \ln 2}{\bar{\gamma}_{k, m}}-\frac{1}{\bar{R}_{k}^{\left(\alpha_{f}\right)}} .
$$

If some of the individual rate constraints are exceeded, the corresponding $\lambda_{k}$ is equal to zero.

Substituting (27) into (25) we obtain

$$
L_{\gamma}^{*}(\mu)=\max _{\lambda_{k}} L_{\gamma}^{*}\left(\lambda_{k}, \mu\right)=\frac{R_{k}}{\bar{R}_{k}^{\left(\alpha_{f}\right)}}-\frac{2^{R_{k}} \mu}{\bar{\gamma}_{k, m}}+\frac{\mu}{\bar{\gamma}_{k, m}}+\mu P_{\text {tot }} .
$$

We next consider the optimization of $L \gamma^{*}(\mu)$ over $\mu$. The function $L \gamma^{*}(\mu)$ can be shown to be a convex function of $\mu$, which can then be minimized via a one-dimensional search with geometric convergence. The optimal values $\mu^{*}$

Correspond to the ones that satisfy the total power constraint (with equality).

We can conclude that, if $G$, are all different, then a given sub channel $\mathrm{m}$ is exclusively assigned to the user $k_{m}^{*}$ satisfying 


$$
\tilde{k}_{m}^{*}=\arg \max _{k} G_{k, m}\left(\tilde{p}_{k, m}^{*}\right)
$$

where $\tilde{p}_{k, m}^{*}$ is the optimal power allocation given by

\subsection{Relative Duality Gap}

$$
\begin{aligned}
\tilde{p}_{k, m}^{*} & =\frac{\bar{R}_{k}^{\left(-\alpha_{f}\right)}+\lambda_{k}^{*}}{\mu^{*} \ln 2}-\frac{1}{\bar{\gamma}_{k, m}}, \quad \text { if } k=\tilde{k}_{m}^{*} \\
& =0, \quad \text { if } k \neq \tilde{k}_{m}^{*} .
\end{aligned}
$$

the relative duality (optimally) gap which indicates how far we are from the optimal value can be expressed as

$$
d^{*}=\frac{g^{*}-f^{*}}{f^{*}} \geq 0
$$

where $f^{*>} 0$ and $g^{*>} 0$ given in (5) and (24) are the optimal values of the primal and dual problems. The inequality follows from the positivity of.f* and the weak duality theorem (D.P. Bertsekas, 1999).

Without the minimum rate constraints in (5), problem (5) becomes a standard convex optimization problem, and then the duality gap is zero. Due to the nonlinearity of the Minimum rate constraints, the convexity of problem (5) does not hold. However, the no convex optimization problem (5) for the investigated OFDMA-based WIMAX system fulfills the time-sharing condition as defined in (W. Yu and R. Lui, 2006). Then when the power constraint is met tightly, that is, with equality, the duality gap is zero, and thus solving the dual problem (24) also solves the primal problem (5).

\subsection{Instantaneous Resource Allocation Based on CSIT.}

In order to assert the relevance of our approach, it was decided to compare it to the instantaneous allocation based on partial CSIT and to the instantaneous allocation based on perfect CSIT.

\subsubsection{Resource Allocation Based on Partial CSIT.}

Assuming that partial channel state information is available at the transmitter in the form of an estimate of the SNR, it has been Shown that resources can be optimally allocated based on this partial CSIT. Let $\gamma_{k, m}$ and

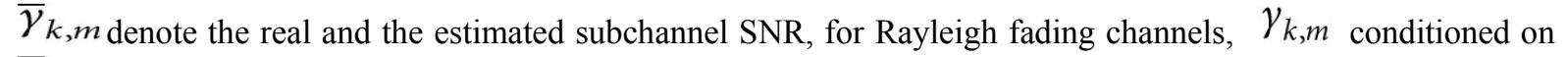
$\bar{\gamma}_{k, m}$, is a noncentral chi-squared distributed random variable with two degrees f freedm (Y. Yao and G. B. Giannakis, 2005)( F. Brah, L. Vandendorpe, and J. Louveaux, 2008). Its probability density function (pdf0 can be approximated to a Gamma function as

$$
T_{\gamma_{k, m}}\left(\gamma_{k, m} \mid \hat{\gamma}_{k, m}\right) \approx \frac{\beta^{\alpha}}{\Gamma(\alpha)} \gamma_{k, m}^{(\alpha-1)} e^{-\beta \gamma_{k, m}}
$$

Under the partial CSIT assumption, the optimization goal is to maximize the expected weighted-sum rate instead of the argotic weighted-sum rate. In(F. Brah, L. Vandendorpe, and J. Louveaux, 2008), the problem has been formulated as

$$
\max _{p_{k, m}, \rho_{k, m}} E_{\gamma} \sum_{k=1}^{K} \sum_{m=1}^{M} \frac{\rho_{k, m}}{\bar{R}_{k}^{\alpha_{f}}} \log _{2}\left(1+p_{k, m} \gamma_{k, m}\right) \mid \hat{\gamma}_{k, m}
$$

Subject to

$$
\begin{gathered}
E_{\gamma} \sum_{m=1}^{M} \rho_{k, m} \log _{2}\left(1+p_{k, m} \gamma_{k, m}\right) \mid \hat{\gamma}_{k, m} \geq R_{k}, \\
\sum_{k=1}^{K} \sum_{m=1}^{M} p_{k, m} \leq P_{\text {tot. }}
\end{gathered}
$$

Using the pdf (32) and applying the KKT optimality conditions, it has been shown in (F. Brah, L. Vandendorpe, 
and J. Louveaux, 2008) that the optimal power allocation $p_{k, m}^{*}$ is the solution of

$$
\frac{\beta^{\alpha}}{\Gamma(\alpha)}{ }_{0}^{\infty} \frac{\gamma_{k, m}^{\alpha}}{1+p_{k, m}^{*} \gamma_{k, m}} e^{-\beta \gamma_{k, m}} d \gamma_{k, m}-\frac{\mu \ln 2}{A_{k}}=0
$$

Also by KKT optimality conditions, it has been shown in (F. Brah, L. Vandendorpe, and J. Louveaux, 2008) that a given subchannel $m$ is exclusively assigned to the user $k_{m}^{*}$ satisfysing

$$
k_{m}^{*}=\arg \max _{k} A_{k} G_{k}\left(p_{k, m}^{*}\right),
$$

Where

$$
\begin{array}{r}
G_{k}\left(p_{k, m}\right)=\frac{\beta^{\alpha}}{\Gamma(\alpha)}{ }_{0}^{\infty} \gamma_{k, m}^{(\alpha-1)} \log _{2}\left(1+p_{k, m} \gamma_{k, m}\right) \\
\times e^{-\beta \gamma_{k, m}} d \gamma_{k, m}-\mu p_{k, m} .
\end{array}
$$

3.4.2 Resource Allocation Based on Perfect CSIT.

Under the unrealistic perfect CSIT assumption, instead of maximizing the argotic or the expected weighted-sum rate, the optimize- tin goal is to maximize the instantaneous weighted-sum rate

$$
\max _{p_{k, m}, \rho_{k, m}} \sum_{k=1}^{K} \frac{1}{\bar{R}_{k}^{\alpha_{f}}} \sum_{m=1}^{M} \rho_{k, m} \log _{2}\left(1+p_{k, m} \gamma_{k, m}\right),
$$

Subject to

$$
\begin{gathered}
\sum_{m=1}^{M} \rho_{k, m} \log _{2}\left(1+p_{k, m} \gamma_{k, m}\right) \geq R_{k}, \\
\sum_{k=1}^{K} \sum_{m=1}^{M} p_{k, m} \leq P_{\text {tot }} .
\end{gathered}
$$

From the KKT optimality conditions, the optimal power allocation, solution of (39) is given by

$$
\tilde{p}_{k, m}^{*}=\frac{A_{k}}{\mu \ln 2}-\frac{1}{\gamma_{k, m}}+
$$

This is a multilevel water-filling power allocation with cut- of subchannel SNR $(\mu \ln 2) / A_{k}$. The difference between (40) and (19) is that the power allocation in (40) depends on the instantaneous subchannel SNR $\gamma \mathrm{km}$ while the one in (19) depends on the mean of the SNR distribution $\gamma_{\mathrm{km}}$ We also deduce from KKT optimality conditions (F. Brah, L. Vandendorpe, and J. Louveaux, 2008) that a given subchannel $m$ is exclusively assigned to the user

$$
\begin{aligned}
& \widetilde{k}_{m}^{*}\left(\rho_{\tilde{k}_{m}^{*}, m}^{-}=1, \rho_{k, m}=0 \text { for } k \neq \tilde{k}_{m}^{*}\right) \text { satisfying } \\
& \tilde{k}_{m}^{*}=\max _{k} A_{k}\left(\log _{2}\left(1+p_{k, m}^{*} \hat{\gamma}_{k, m}\right)-\mu p_{k, m}^{*}\right)
\end{aligned}
$$

\section{Simulation Results}

In Figure 1, the performance of the proposed adaptive resource allocation is compared to those of optimal resource allocation based on perfect CSIT, resource allocation based on partial CSIT and a uniform power allocation. The result shows that the proposed adaptive resource allocation brings significant gain over resource allocation based on partial CSIT with higher estimation error. We can observe that when $\gamma_{e} / n$ is small, that means the effect of the estimation error is less dominant than the one of the background noise, the optimization under partial CSI is closed to the one under perfect CDIT. For very low estimation errors, the partial CSIT-based scheme outperforms the perfect CDIT scheme. The weighted-sum rate degrades quickly as the Estimation error grows, especially for high SNRs. The highest weighted-sum rate is obtained with perfect CSIT but the difference 
in terms of performance is not so significant compared to the difference of complexity between CDIT-Based and CSIT-based allocation schemes. The proposed method outperforms the uniform power allocation.

Figure 2 shows the user's rate for different allocation schemes when the users minimum data rate demands are constrained to $R_{3} \quad 2_{2} R_{2} \quad 3 R_{1}$ and the fairness parameter $\alpha_{f}$ is set to 0 . We observe that under optimal allocation based on perfect CDIT, the need of all users in terms of data rate is satisfied. This is neither the case under allocation based on partial CSIT with high estimation error nor under uniform

Allocation where the high data rate demand of user 3 is not satisfied.

Figure 3 illustrates the tradeoff between the maximized weighted-sum rate and the fairness constraint when the minimum rate demand is relaxed to $\left(1-\eta_{q}\right) R k_{k}$ with $0 \leq \mathrm{N}<=0.8$. The average user rates are updating according to (4) with $\mathrm{Rn}=20$.

\section{Conclusion}

In this paper, we have presented a resource allocation method that maximizes the argotic weighted-sum rate of a multiuser Mobile WiMAX while satisfying user's specific minimum rate demand and system fairness requirement for a given power budget. Though this is originally a nonlinear optimization problem, the problem can be reformulated as a Lagrangian dual problem. From this, a method has been proposed to Efficiently solve the problem. The proposed method can find the optimal solution with significant lower computational complexity than the optimal CSIT-based allocation schemes. In fading environments, even with CDIT only, adaptive resource allocation strategies provide performance gain for OFDMA systems. Since user mobility is the principal driving force for mobile WiMAX, CDIT-based resource allocation

Strategies are of particular interest. These methods can be applied to other mobile OFDMA-based wireless systems such as Long Term Evolution (LTE) or High-Speed Downlink Packet Access (HSDPA).

\section{References}

C. T. K. Ng and A. J. Goldsmith. (2006). Capacity and power allocation for transmitter and receiver cooperation in fading channels, in Proceedings of IEEE International Conference on Communications (ICC '06), vol. 8, pp. 3741-3746, Istanbul, Turkey, July 2006.

C. Y. Wong, C. Y. Tsui, R. S. Cheng and K. B. Letaief. (1999). A real-time sub-carrier allocation scheme for multiple access downlink OFDM transmission, in Proceedings of the 50th IEEE Vehicular Technology Conference (VTC '99), vol. 2, pp. 1124-1128, Amsterdam, the Netherlands, September 1999.

D. Kivanc, G. Li and H. Liu. (2003). Computationally efficient bandwidth allocation and power control for OFDMA, IEEE Transactions on Wireless Communications, vol. 2, no. 6, pp. 1150-1158, 2003.

D.P. Bertsekas. (1999). Nonlinear Programming, Athena Scientific, Boston, Mass, USA, 2nd edition, 1999.

F. Brah, L. Vandendorpe, and J. Louveaux. (2008). Constrained resource allocation in OFDMA downlink systems with partial CSIT, in Proceedings of IEEE International Conference on Communications (ICC '08), pp. 4144-4148, Beijing, China, May 2008.

I. C. Wong, Z. Shen, B. L. Evans, and J. G. Andrews. (2004). A low complexity algorithm for proportional resource allocation In OFDMA systems, in Proceedings of IEEE Workshop on Signal Processing Systems (Sips '04), pp. 1-6, Austin, Tex, USA, October 2004.

M. Abramowitz and I. Stegun. (1964). Handbook of Mathematical Functions with Formulas, Graphs, and Mathematical Tables, Dover, New York, NY, USA, 1964.

M. Ergen, S. Coleri and P. Varaiya. (2003). Qos aware adaptive resource allocation techniques for fair scheduling in OFDMA Based broadband wireless access systems, IEEE Transactions on Broadcasting, vol. 49, no. 4, pp. 362-370, 2003.

N. Damji and T. Le-Ngoc. (2006). Adaptive downlink resource allocation strategies for real-time data services in OFDM cellular systems, EURASIP Journal on Wireless Communications and Networking, vol. 2006, Article ID 17526, 11 pages, 2006.

Recommendation ITU-R M.1225. (1997). Guidelines for evaluation of radio transmission technologies for IMT-2000.

S. Boyd and L. Vandenberg he. (2004). Convex Optimization, Cam- bridge University Press, Cambridge, UK, 2004.

S. Pietrzyk. (2006). OFDMA for Broadband Wireless Access, Artech House, London, UK. 
T. Sartenaer, J. Louveaux and L. (2008). Vandendorpe, Balanced capacity of wire line multiple access channels with individual Power constraints, IEEE Transactions on Communications, vol. 56, no. 6, pp. 925-936, 2008.

T. Sartenaer, L. Vandendorpe and J. Louveaux. (2005). Balanced capacity of wire line multiuser channels, IEEE Transactions on Communications, vol. 53, no. 12, pp. 2029-2042, 2005.

W. Yu and R. Lui. (2006). Dual methods for nonconvex spectrum optimization of multicarrier systems. IEEE Transactions on Communications, vol. 54, no. 7, pp. 1310-1322.

WiMAX Forum. (2006). Mobile WiMAX - part I: a technical overview and performance evaluation, March 2006.

WiMAX Forum. (2007). A comparative analysis of mobile WiMAX deployment alternatives in the access networks, May 2007.

X. Zhang, E. Zhou, R. Zhu, S. Liu, and W. Wang. (2005). Adaptive multiuser radio resource allocation for OFDMA systems, in Proceedings of IEEE Global Telecommunications Conference (GLOBECOM '05), vol. 6, pp. 3846-3850, St. Louis, Mo, USA, November 2005.

Y. Yao and G. B. Giannakis. (2005). Rate-maximizing power allocation in OFDM based on partial channel knowledge, IEEE Transactions on Wireless Communications, vol. 4, no. 3, pp. 1073-1083, 2005.

Z. Han, Z. Ji, and K. J. R. Liu. (2005). Fair multiuser channel allocation for OFDMA networks using Nash bargaining solutions and coalitions, IEEE Transactions on Communications, vol. 53, no. 8, pp. 1366-1376, 2005.

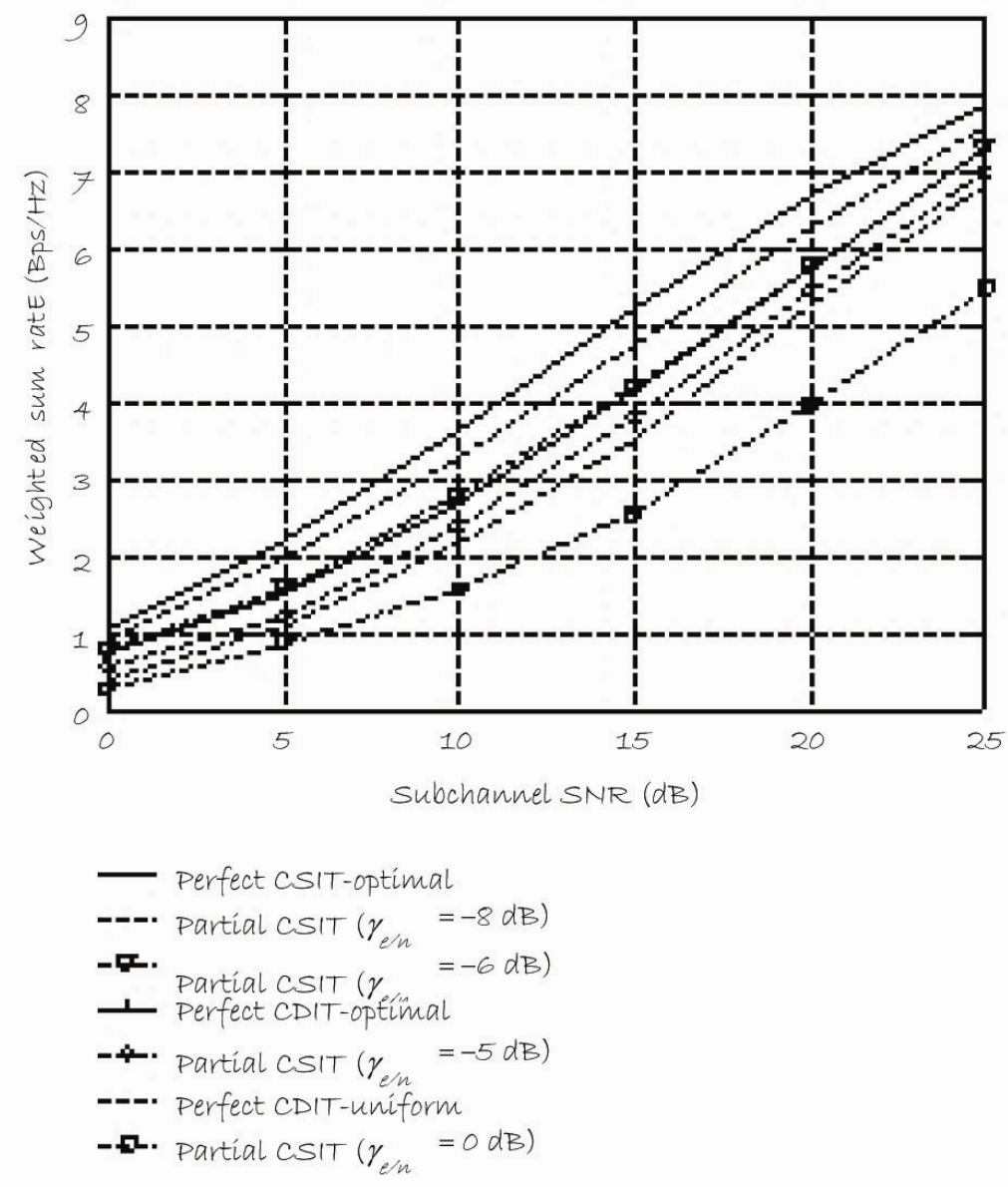

Figure 1. Maximized weighted-sum rate versus mean SNR for dif- Ferment resource allocation schemes and fairness parameter $\alpha_{\text {.f }}=0$. 


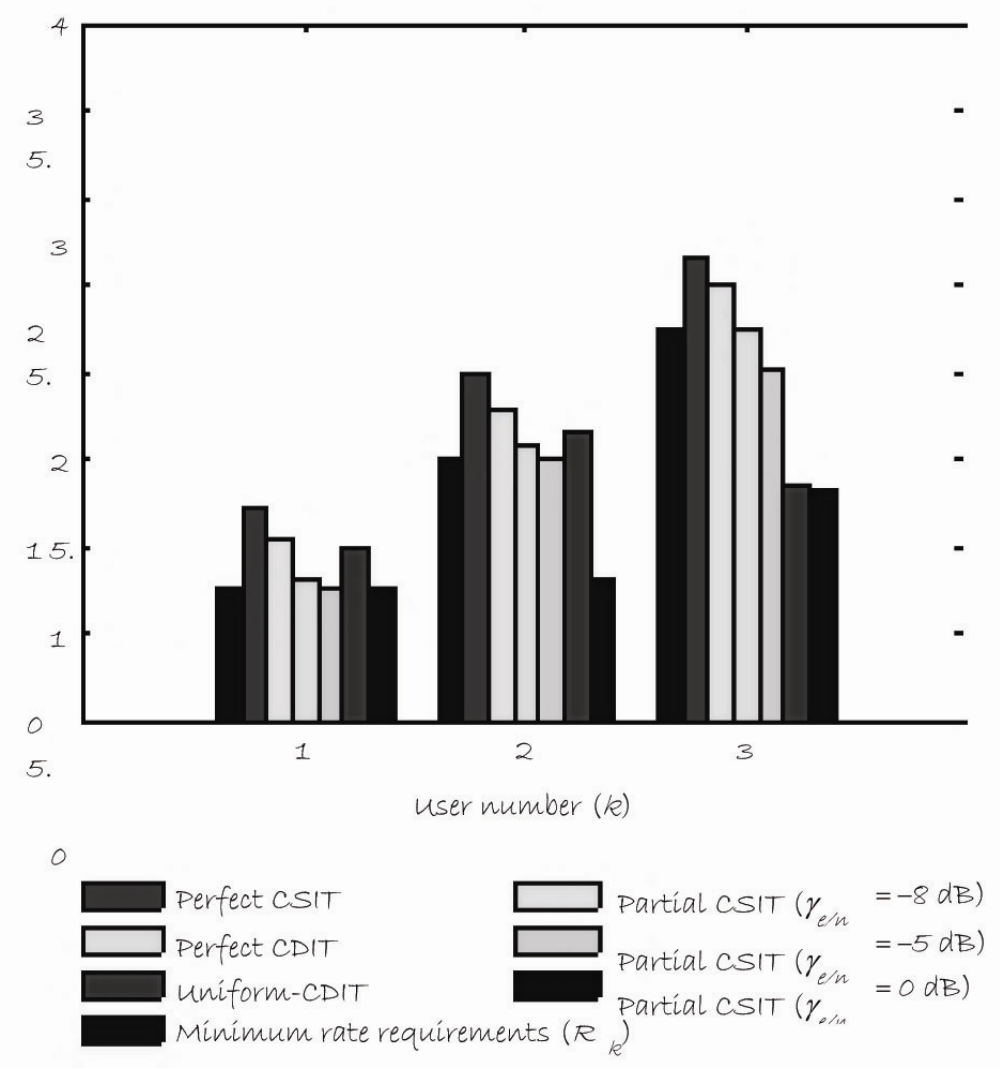

Figure 2. Users rates for different resource allocation schemes, Mean SNR of $15 \mathrm{~dB}$ and fairness parameter $\alpha_{. f}=0$.



Figure 3. Tradeoff Between maximized weighted-sum rate and Fairness requirement for different resource allocation schemes and Mean SNR of $15 \mathrm{~dB}$. 


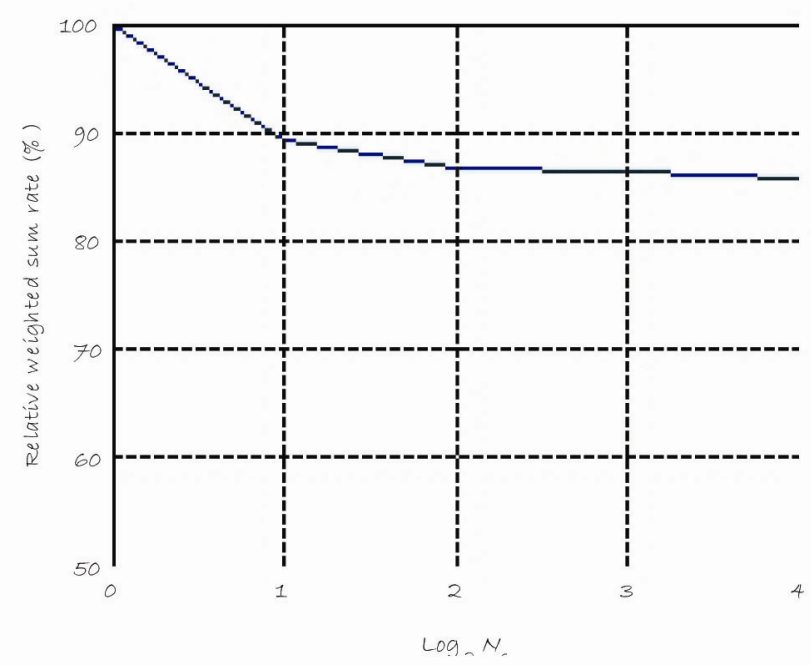

Figure 4. Tradeoff between reduced complexity and performance Degradation of the CDIT-based allocation compared with the Perfect CSIT. 